\section{Being Sensitive: to Specify When Amino Acid Tracers Accumulate in a Brain Lesion}

TO THE EDITOR: I read with considerable interest the recent report by Rapp et al. (1) demonstrating the diagnostic performance of $O$-(2- ${ }^{18} \mathrm{~F}$-fluoroethyl)-L-tyrosine $\left({ }^{18} \mathrm{~F}-\mathrm{FET}\right)$ PET in newly diagnosed cerebral lesions suggestive of glioma. This retrospective but nevertheless convincing study provides us with substantial data on the accumulation of an amino acid PET tracer in glioma for a relatively large patient cohort. As the authors emphasize, their study meets the criteria of strict standardization of PET acquisition protocols. Considering that the clinical value of amino acid brain PET imaging for differential diagnosis still can be considered a work in progress, these precisely documented findings must not be underestimated.

On the other hand, the quintessence of these evaluations can be found in previous publications, and of course this fact supports the merit of the data of Rapp et al. in showing that high-grade glioma nearly always exhibits intense accumulation of ${ }^{18} \mathrm{~F}$-FET and that low uptake therefore excludes a high-grade tumor with high probability. Also, their finding of higher levels of ${ }^{18} \mathrm{~F}$-FET uptake in high-grade glioma than in low-grade glioma is not new, and because of their observed marked overlap in uptake quantification-again supporting previous data - the authors' conclusion that ${ }^{18} \mathrm{~F}$-FET uptake ratios provide valuable additional information for grading of gliomas may be questionable at least in clinical practice.

We also face the substantiality that on visual rating about two thirds of low-grade glioma are ${ }^{18} \mathrm{~F}$-FET-positive and one third is ${ }^{18} \mathrm{~F}$ FET-negative. A study by our group published in 2010 (2) is cited by Rapp et al. as "the currently largest series" of patients. Additionally, they comment that "these results, however, were based only on a visual rating, and histology was available in only two thirds of patients." This is right, as the images were of course rated visually by the reporting physicians. But we also published a lesion-to-brain ratio-correlated to histology when available-with results comparable to the findings of our German colleagues.

Here comes my main message: evidentially, it is important to have valuable data on amino acid uptake in lesions that are very suggestive of glioma and subsequently proven to be glioma on pathologic examination. But we have to look forward. What is the nature of ${ }^{18} \mathrm{~F}$-FET uptake in lesions that are possibly, but not very probably, glioma? Published data may focus on observational studies as well, as shown by the recent work of Hutterer et al. (3), in which only three quarters of patients had histology available. For the individual patient, the valuable information obtained from ${ }^{18}$ F-FET PET - additional to that from MR imaging in general - would allow for better decisions about medical management. We must know more about possible pitfalls, such as whether ${ }^{18}$ F-FET accumulates in abscesses, multiple sclerosis plaque, vasculitis (3), or radiation-induced astrogliosis (4). Then, we can make comparisons with a typical profile of ${ }^{18} \mathrm{~F}$-FET uptake in glioma as shown by the retrospective data of Rapp et al. and others.

COPYRIGHT @ 2013 by the Society of Nuclear Medicine and Molecular Imaging, Inc.
The scientific community should also embrace the concept that negative or low-accumulating lesions-suggestive of low-grade glioma for example, by MR imaging — are related to a good prognosis even without specific therapy. Even more data are missing related to brain metastases from solid tumors, such as the already helpful data provide by Langen's group (5).

\section{REFERENCES}

1. Rapp M, Heinzel A, Galldiks N, et al. Diagnostic performance of ${ }^{18}$ F-FET PET in newly diagnosed cerebral lesions suggestive of glioma. J Nucl Med. 2013;54:229235

2. Pichler R, Dunzinger A, Wurm G, et al. Is there a place for FET PET in the initial evaluation of brain lesions with unknown significance? Eur J Nucl Med Mol Imaging. 2010;37:1521-1528.

3. Hutterer M, Nowosielski M, Putzer D, et al. $\left[{ }^{18} \mathrm{~F}\right]$-fluoro-ethyl-L-tyrosine PET: a valuable diagnostic tool in neuro-oncology, but not all that glitters is glioma. Neuro-Oncol. 2013;15:341-351.

4. Pichler R, Wurm G, Nussbaumer K, Kalev O, Silyé R, Weis S. Sarcoidosis and radiation-induced astrogliosis causes pitfalls in neuro-oncologic positron emission tomography imaging by O-(2-[ $\left[{ }^{18} \mathrm{~F}\right]$ fluoroethyl $)-\mathrm{L}-$ tyrosine. J Clin Oncol. 2010;28: e753-e755.

5. Galldiks N, Stoffels G, Filss CP, et al. Role of $O-\left(2-{ }^{18} \mathrm{~F}\right.$-fluoroethyl)-L-tyrosine PET for differentiation of local recurrent brain metastasis from radiation necrosis. J Nucl Med. 2012;53:1367-1374.

\section{Robert Pichler}

Wagner-Jauregg Hospital

Wagner-Jauregg Weg 15

A-4021 Linz, Austria

E-mail: robert.pichler@gespag.at

Published online Mar. 27, 2013.

DOI: 10.2967/jnumed.113.122325

REPLY: We agree with Dr. Pichler that our report on $O-\left(2-{ }^{18} \mathrm{~F}-\right.$ fluoroethyl)-L-tyrosine ( ${ }^{18} \mathrm{~F}$-FET) uptake in primary brain lesions is in line with previous studies and we have cited the corresponding literature correctly. There is no need to point out that fact once again. In comparison with previous studies, the prominent features of our study are the larger size of the patient group, histologic confirmation in nearly all cases, and a clear and reproducible technique of ${ }^{18}$ F-FET uptake evaluation. Visual evaluations of ${ }^{18} \mathrm{~F}$-FET PET scans are subjective and may be difficult for inexperienced physicians. Therefore, the guidelines of the European Association of Nuclear Medicine and German Society of Nuclear Medicine for brain tumor imaging using labeled amino acid analogs recommend the use of a threshold value of the lesion-to-brain ratio to distinguish a positive result from nonspecific amino acid uptake $(1,2)$. Our report provides threshold values of ${ }^{18} \mathrm{~F}$-FET uptake for primary brain lesions that are essential for clinical decision making (3).

We also agree with Dr. Pichler that knowledge of the mechanisms leading to increased ${ }^{18} \mathrm{~F}$-FET uptake in nonneoplastic brain lesions is important. We have undertaken several experimental studies of ${ }^{18}$ F-FET uptake in animal models of cerebral infarctions, abscesses, and hematoma (4-6). Those studies demonstrated that increased ${ }^{18}$ F-FET uptake temporarily occurred in areas with 
reactive astrocytosis but not in macrophage infiltration or activated microglia. In humans, the histologic finding of pronounced reactive astrocytosis was confirmed in different nonneoplastic lesions that exhibited increased ${ }^{18} \mathrm{~F}$-FET uptake $(7,8)$. Thus, according to the current knowledge, a high incidental uptake of ${ }^{18} \mathrm{~F}-\mathrm{FET}$ in benign brain lesions is most likely due to reactive astrocytosis.

Furthermore, in a clinical study we already addressed the problem of nonspecific brain lesions on MR imaging with low ${ }^{18} \mathrm{~F}$-FET uptake (9). We observed that normal or low ${ }^{18} \mathrm{~F}-\mathrm{FET}$ uptake is a strong predictor for a benign course, with the eventual development of a low-grade glioma.

We would like to emphasize that the data on lesion-to-brain ratios of ${ }^{18} \mathrm{~F}$-FET uptake in different brain lesions at initial diagnosis may be helpful for decision making but that the additional value of ${ }^{18} \mathrm{~F}$-FET PET lies in defining an optimal site for biopsy and determining the extent of metabolically active tumor for treatment planning.

\section{REFERENCES}

1. Langen KJ, Bartenstein P, Boecker H, et al. German guidelines for brain tumour imaging by PET and SPECT using labelled amino acids. Nuklearmedizin. 2011; 50:167-173.

2. Vander Borght T, Asenbaum S, Bartenstein P, et al. EANM procedure guidelines for brain tumour imaging using labelled amino acid analogues. Eur J Nucl Med Mol Imaging. 2006;33:1374-1380.
3. Rapp M, Heinzel A, Galldiks N, et al. Diagnostic performance of ${ }^{18}$ F-FET PET in newly diagnosed cerebral lesions suggestive of glioma. J Nucl Med. 2013;54:229-235.

4. Salber D, Stoffels G, Oros-Peusquens AM, et al. Comparison of O-(2-18 F-fluoroethyl)-

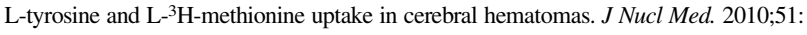
790-797.

5. Salber D, Stoffels G, Pauleit D, et al. Differential uptake of O-(2- ${ }^{18}$ F-fluoroethyl)L-tyrosine, L- ${ }^{3} \mathrm{H}$-methionine, and ${ }^{3} \mathrm{H}$-deoxyglucose in brain abscesses. J Nucl Med. 2007;48:2056-2062.

6. Salber D, Stoffels G, Pauleit D, et al. Differential uptake of $\left[{ }^{18}\right.$ F $]$ FET and $\left[{ }^{3} \mathrm{H}\right] 1-$ methionine in focal cortical ischemia. Nucl Med Biol. 2006;33:1029-1035.

7. Floeth FW, Pauleit D, Sabel M, et al. ${ }^{18}$ F-FET PET differentiation of ringenhancing brain lesions. J Nucl Med. 2006;47:776-782.

8. Pichler R, Wurm G, Nussbaumer K, Kalev O, Silyé R, Weis S. Sarcoidois and radiationinduced astrogliosis causes pitfalls in neuro-oncologic positron emission tomography imaging by O-(2-[18 F]fluoroethyl)-L-tyrosine. J Clin Oncol. 2010;28:e753-e755.

9. Floeth FW, Sabel M, Stoffels G, et al. Prognostic value of ${ }^{18}$ F-fluoroethylL-tyrosine PET and MR imaging in small nonspecific incidental brain lesions. J Nucl Med. 2008;49:730-737.

\section{Karl-Josef Langen}

Institute of Neuroscience and Medicine, Forschungszentrum Jülich Department of Nuclear Medicine, Aachen University Clinic

Wilhelm-Johnen-Strasse

D-52425 Jülich, Germany

E-mail: k.j.langen@fz-juelich.de

Published online Mar. 27, 2013.

DOI: 10.2967/jnumed.113.122960

\section{Erratum}

In the article "Assessment of Cellular Proliferation in Tumors by PET Using ${ }^{18}$ F-ISO-1," by Dehdashti et al. ( $\mathrm{J}$ Nucl Med. 2013;54:350-357), the name of the tenth author in the byline was misspelled. The correct name is Nina Wagner-Johnston. The authors regret the error. 